\title{
MedienPädagogik
}

Zeitschrift für Theorie und Praxis der Medienbildung www.medienpaed.com

Themenheft Nr. 28: Tagungsband: Bildung gemeinsam verändern: Diskussionsbeiträge und Impulse aus Forschung und Praxis. Herausgegeben von David Meinhard, Valentin Dander, Andrea Gumpert, Christoph Rensing, Klaus Rummler und Timo van Treeck.

\section{(Portale), die zum Nachdenken anregen Einblicke in eine Interfaceanalyse des Augmented Reality Spiels Ingress}

Franco Rau

\begin{abstract}
Zusammenfassung
Augmented Reality Spiele wie 〈Ingress〉 und 〈PokémonGo〉 stellen aktuell neue mediale Phänomene dar. Eine zentrale Differenz zu klassischen Massively Multiplayer Online Games (MMOs) ist die Verzahnung von virtuellen und realen Objekten als konstitutive Spielelemente. In Anknüpfung an die Perspektive, digitale Spiele als Kultur- und Bildungsräume zu verstehen, widmet sich der Beitrag der Frage, inwiefern das Ingress-Spielen die Perspektive von Spieler/innen auf den öffentlichen Raum verändern kann. In erster Annäherung an diese Fragestellung wird auf Basis einer Interfaceanalyse gezeigt, wie ‘Portale - virtueller Repräsentationen realer Objekte im Spiel - potenziell Irritationen und Differenzerfahrungen ermöglichen. Diese können zum Ausgangspunkt weiterführender Reflexionen werden. Dafür werden exemplarisch 〈Portale〉 diskutiert, die (1.) weiterführende Informationen zu realen Objekte bieten, die (2.) Momentaufnahmen nicht mehr existierender kultureller Objekte darstellen und die (3.) reale Objekte in ironischer Weise präsentieren.
\end{abstract}

Portals Encouraging Reflection. Insights into an Interface Analysis of the Augmented Reality Game Ingress

\begin{abstract}
Augmented reality games like 〈Ingress〉 or 〈PokémonGo〉 illustrate new media phenomena. The central deviation to Massively Multiplayer Online Games (MMOs) is the indentation of virtual and real objects as constitutive elements of the game. This article focuses on the question, to what extent playing Ingress may lead to a modification of the players' perspective on public space. Based on an interface analysis, this paper shows how 〈portals - virtual representations of real objects within the game - potentially offer moments of irritation and experiences of difference. These could be the starting point for further reflection. In order to demonstrate this possible process, this paper will discuss ‘portals〉, which (1.) offer further information on real objects, (2.) represent snapshots of no longer existent cultural objects, or (3.) present real objects in an ironic manner.
\end{abstract}




\section{Zur Diskussion von digitalen Spielen}

Computerspiele bzw. digitale Spiele werden in der Öffentlichkeit noch sehr kontrovers diskutiert, wie bei jüngeren Amokläufen sichtbar wurde. Eine Bewertung und Analyse von Computerspielen ${ }^{1}$ auf die Darstellung von Gewalt zu reduzieren, wird den vielfältigen Phänomenen digitaler Spiele jedoch in keiner Weise gerecht (vgl. Beil 2013, Breuer und Elson 2015, Fromme et al. 2008). In Betrachtung aktueller Publikationen zeigt sich vielmehr, wie vielfältig digitale Spiele sowie das digitale Spielen als Forschungsgegenstand konzeptionalisiert und analysiert werden (vgl. Beil 2013, Freyermuth et al. 2013, Kaminski und Lorber 2010). In der pädagogischen Diskussion von digitalen Spielen lassen sich dabei zwei zentrale Diskussionsstränge und Betrachtungsweisen von Spielen unterschieden:

1. digitale Spiele als Vermittlungsinstrumente bzw. als Serious Games (vgl. Ganguin und Hoblitz 2013, Gotto 2013, Pfannstiel, Sänger und Schmidt 2009) sowie

2. digitale Spiele als «Kultur- und Bildungsräume» (vgl. Fromme et al. 2008, Fromme und Könitz 2014, Grell und Nuss 2010).

In der Betrachtung von digitalen Spielen als Serious Games werden diese als Instrumente zur Erreichung spezifischer Ziele verstanden: Mit ihnen soll «etwas erreicht, trainiert und gelernt werden» (Gotto 2013, 139). Sie dienen als Ergänzung pädagogischer Vermittlungssituationen, als «Türöffner» oder zur «Belohnung und Verstärkung» (Fromme et al. 2010, 43), oder stellen Versuche dar, pädagogisch relevant erachtete Inhalte spielerisch zu vermitteln (vgl. Fromme et al. 2010, 43). Demgegenüber steht die Perspektive, digitale Spiele als Kultur- und Bildungsraum zu begreifen, welche im Fokus dieses Artikels steht. Computerspiele eröffnen in diesem Verständnis neue Erfahrungsräume und bieten Optionen zur sozialen Kommunikation und Interaktion (vgl. Fromme et al. 2008). Grell und Nuss (vgl. 2010, 222) vertreten in diesem Zusammenhang die Annahme, dass im Rahmen dieser interaktiven Räume Differenzerfahrungen möglich werden, welche Anlässe für Reflexionen und Bildungsprozesse sein können. Differenzerfahrungen sind für Grell und Nuss (2010) im Selbst- und Fremderleben möglich. Spielende Personen sind beim Spielen, so Grell und Nuss (2010, 222), «mit einer fremden (virtuellen) Welt konfrontiert, deren Regeln und Möglichkeiten sie erst kennenlernen müssen». Im Gegensatz zu vielen anderen Unterhaltungsmedien sind sie nicht nur Rezipienten/innen, sondern handeln selbst interaktiv in der Spielumgebung. «Erfahrungen von Fremdheit» sowie das Scheitern von Handlungsstrategien können als Differenzerfahrungen verstanden werden. Potenziell erfahrbar sind diese Differenzen für Grell und Nuss $(2010,222)$ u. a. auf der Spielinteraktionsebene sowie auf der inhaltlichen Ebene, z. B. in Form eines moralischen Dilemmas. Ferner argumentiert Fromme (2006, 201), dass bei Computerspielen auch «(visuelle) Gestaltungsmittel anzutreffen sind, die einen in diesem Sinne irritierenden Charakter

1 Computerspiele und digitale Spiele werden im Folgenden synonym verwendet und dienen als Sammelbegriff für Spiele für Konsolen, Computer und mobile Endgeräte. 
haben». Er spricht in diesem Kontext von der «Möglichkeit einer Art immersiver Reflexivität» (ebd., 201).

Das Verständnis von digitalen Spielen als Kultur- und Bildungsräume meint jedoch nicht, dass die sich virtuell eröffnenden Erfahrungsräume von der realen Welt abgeschlossen oder gar isoliert zu betrachten sind. Die zunehmende «technische Durchdringung des sozialen und kulturellen Lebens» führe vielmehr, so Fromme et al. (2008), zu einer Vermischung von virtuellen und realen Erfahrungsräumen. Bei Augmented Reality Spielen wie Ingress, verschwimmt die Grenze von virtuellen und realen Erfahrungsräumen nicht nur im Rahmen der Spielgemeinschaften und Spielkulturen. Vielmehr wird die Kombination virtueller und realer Erfahrungsräume - wie im Verlauf des Beitrages exemplarisch gezeigt wird - zum konstitutiven Bestandteil des Spiels.

Am Arbeitsbereich «Allgemeine Pädagogik und Medienpädagogik» der TU Darmstadt wurde 2015 das Forschungsprojekt «Zwischen Links, Portalen und Kultur» gestartet. Inwiefern Ingress das Potenzial besitzt, Differenzerfahrungen und Reflexionsanlässe in der Wahrnehmung des (lokalen) öffentlichen Raums zu ermöglichen, wird in diesem Beitrag anhand ausgewählter Interfaceelemente diskutiert.

\section{Augmented Reality Spiele und die neuen Erfahrungsräume von Ingress}

Der Begriff «Augmented Reality» kann frei als «erweiterte Realität» übersetzt werden. Damit ist gemeint, so Damberger $(2016,1)$, «dass die realen Dinge, die wir um uns herum wahrnehmen, um virtuelle Zusatzinformationen erweitert werden.» Einen Zugang zu diesen Informationen bieten Smartphones bzw. Smartphone-Apps. Über die Betrachtung bestimmter Objekte durch die Kamera oder über die Positionsbestimmung des Standortes können entsprechende Applikationen Informationen in verschiedenen Varianten darstellen: von der Präsentation virtueller Möbel in der eigenen Wohnung, über das Anzeigen von Informationen für Touristen/innen beim Betrachten von Sehenswürdigkeiten bis hin zur Darstellung der aktuell sehr beliebten Pokémon bei PokémonGo (2016). Im Spiel Ingress finden sich diese zusätzlichen Informationen bei so genannten ‘Portalen〉. Dabei handelt es sich um kulturträchtige Orte oder Objekte wie beispielsweise historische Gebäude, öffentliche Plätze oder moderne Strassenkunst. Die im Spiel Ingress (2013) sichtbaren Portale basieren überwiegend auf von Spieler/innen eingereichten - und von Niantic begutachteten - Vorschlägen. Welche Orte und konkreten Objekte ein virtuelles Portal darstellen, wird über den «Scanner», d. h. über die jeweiligen mobilen Geräte sichtbar. Das virtuelle Spielfeld von Ingress basiert auf einer Abbildung der realen physischen Welt (Abb. 1). 
Worum geht es bei dem Spiel Ingress und was ist das Neue?

In der Narration von Ingress gelangt eine neuartige Energie bzw. Materie über diese Portale in unsere Welt: Exotic Matter (XM). Zwei Fraktionen spielen weltweit um die Kontrolle dieser XM. Während die eine Seite in der XM neue Chancen für den Fortschritt der Menschheit sieht und diese dafür nutzen möchte («Enlightenment»), betont die andere Fraktion die potenziellen Gefahren und möchte den weiteren Einfluss der XM verhindern («Resistance») ${ }^{2}$. Als Spieler/in muss man sich zu Beginn zwischen diesen zwei gegeneinander antretenden Fraktionen entscheiden. Die Möglichkeit XM für die gewählte Fraktion zu kontrollieren besteht in der Kontrolle der Portale. Das Spiel Ingress beschränkt sich entsprechend nicht auf die Darstellung virtueller Zusatzinformationen, sondern ermöglicht auch Interaktionen mit virtuellen Objekten. Ähnlich dem Spielmodus «Capture the Flag» können Portale eingenommen, mit virtuellen Gegenständen modifiziert oder zur Generierung neuer Spielgegenstände verwendet werden. Das Interessante dabei ist, dass die Spieler/innen zur Übernahme der Kontrolle eines Portals physikalisch vor Ort sein müssen. Statt einen Avatar per Mausklick, Bewegungstasten oder Controller zu steuern, erfolgt die Erfassung von Bewegungen bei Ingress über die Standortdaten der mobilen ‘Spielgeräte). Zum Spielen von Ingress müssen sich Spieler/innen entsprechend physisch durch ihre lokale Umgebung, durch ihre Nachbarschaft oder ihre gesamte Stadt bewegen. Für grössere Spielaktionen, die ganze Städte und Länder umfassen können, ist die Koordination von vielen Spielern/-innen an unterschiedlichen Orten notwendig. Diese Notwendigkeit, sich zeitlich abgestimmt an physisch verschiedenen Orten zu bewegen, um virtuell miteinander spielen zu können, ist ein neues Spezifikum von Augmented Reality-Spielen und markiert einen zentralen Unterschied zu klassischen Massively Multiplayer Online Games.

2 Die Enlightenment-Fraktion, die im Spiel grün visualisiert ist, wird im Rahmen der Spielcommunity häufig als «Frösche» benannt. Die Resistance-Fraktion, die im Spiel blau visualisiert ist, wird im Rahmen der Spielcommunity als «Schlümpfe» bezeichnet. 

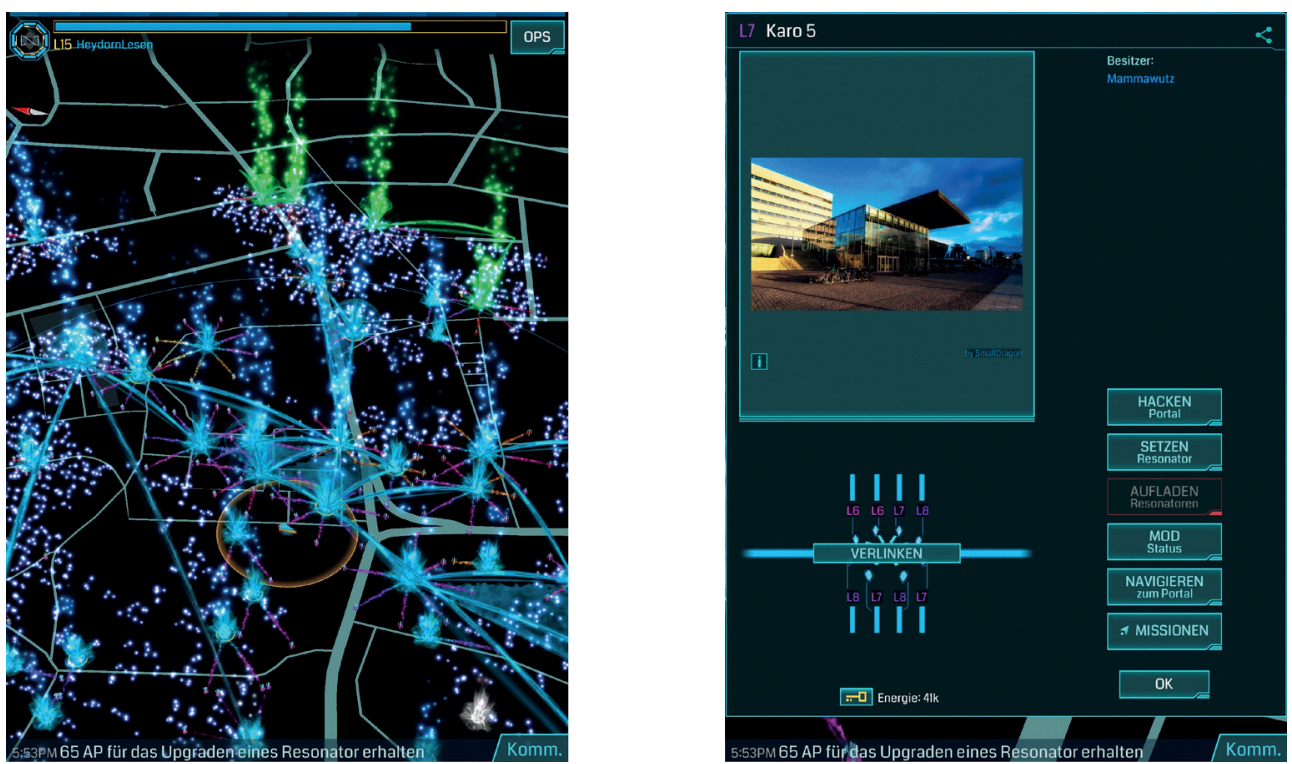

Abb. 1.: Ansicht der TU Darmstadt (Campus Mitte) durch den Scanner (links) sowie die Detailansicht des Portals «Karo 5» (rechts). Quelle: Screenshot des Spiels Ingress.

\section{Zur pädagogischen Relevanz und Einblicke in aktuelle Studien}

Die holzschnittartig skizzierten Spiel- und Interaktionsmöglichkeiten geben erste Hinweise darauf, wie im und mit dem Spiel neue Optionen zur sozialen Kommunikation und Interaktion potenziell eröffnet werden. Im Kontext aktueller Forschungsarbeiten zu internetbasierten Gemeinschaften (vgl. Gross et al. 2008, Grell und Nuss 2010) sowie zur Analyse von Bildungspotenzialen digitaler Spiele (vgl. Fromme et al. 2008, Grell und Nuss 2010), erscheinen die folgenden Fragen relevant: Inwiefern können diese Optionen zu (Differenz-)Erfahrungen zwischen den digitalen und realen Objekten führen und welche Bedeutung haben diese neuen Interaktionsmöglichkeiten für die Entwicklung von Online-Gaming-Kulturen? Antworten auf diese Fragen finden sich in aktuellen Forschungsarbeiten zu Ingress jedoch kaum (vgl. Chess, 2014, Majorek und Du Vall 2015, Stingeder 2013). Chess (2014) fokussiert in ihrem Beitrag über Ingress die digitale Erzählung des Spiels. Sie argumentiert, dass Ingress Globalismus mit Regionalismus in einer neuen Art und Weise kombiniert. Stingeder (2013) markiert eine völlig neue Form der Interaktivität und betrachtet insbesondere das Problem des Data-Minings. Über die notwendige Positionsbestimmung der mobilen «Spielgerätes und der Dokumentation relevanter Spielinteraktionen ist ein anonymes Spielen nur begrenzt möglich. Hinsichtlich der Auswirkungen von Ingress äussern sich Majorek und Du Vall (2015) sehr optimistisch: «The game [...] gives people an entirely new perspective on their cities and the environment» (Majorek und Du Vall 2015, 18). Die Autorinnen kommen in ihrer Studie zu dem weitreichenden Fazit: 
Ingress is becoming a tool for new kind of socialization; it helps people return to typical interactions and frees individuals from functioning only in the virtual world. This type of game gives hope of restoring coexistence in the real world and of utilizing new technologies to create true bonds, and not, as feared, to petrify atomized benchmarks and interactions, which are almost entirely disconnected from reality. (Majorek und Du Vall 2015, 19f.)

Die dargestellten Erkenntnisse über Ingress basieren vor allem auf teilnehmenden Beobachtungen und informellen Interviews (Majorek und Du Vall 2015, Stingeder 2013). Die Reichweite der dargestellten Aussagen müssen entsprechend kritisch betrachtet werden. Wenngleich die vorgestellten Arbeiten erste Hinweise auf mögliche Bildungspotenziale bieten, sind Untersuchungen mit einem expliziten Fokus auf die zuvor markierten Fragestellungen bzw. auf Lern- und Bildungsprozesse nicht bekannt und können als Forschungslücke markiert werden.

\section{Portale in Ingress - Neue Perspektiven auf den öffentlichen Raum?}

Zur Annäherung an die Fragestellung «Inwiefern kann das Ingress-Spielen die Perspektive von Spielern/-innen auf den öffentlichen Raum verändern?» wird sich im Folgenden an den methodischen Vorschlägen von Fromme et al. (2008) zur Untersuchung von Computerspielen orientiert. Aus ihrer Perspektive bedarf es (a) einer Analyse der strukturellen Eigenschaften, des Interfaces sowie möglicher Interaktionen des Spiels und (b) einer Untersuchung der zugehörigen Computerspielgemeinschaften:

Unter der visuellen Oberfläche eines Computerspiels bilden seine Tiefenstrukturen - der Handlungsraum, der Coderaum sowie der das Spiel überschreitende soziale Raum - wichtige Bereiche, die bei der Beurteilung und Analyse von Computerspielen beachtet werden müssen. Bildungsaspekte lassen sich generell durch eine Analyse auf diesen Ebenen auffinden, also in den zwei Dimensionen a) der Interaktions- und Interfacestruktur sowie b) der sozialen Interaktionsstruktur im Umfeld des Spiels. (Fromme et al. 2008, 18)

Unter Berücksichtigung des begrenzten Umfangs beschränkt sich der vorliegende Beitrag auf die Analyse der ersten Dimension. Im Sinne von Fromme $(2006,201)$ wird der Analysefokus auf «die Immersion irritierenden Gestaltungsmittel» bzw. Interfaceelemente gerichtet. Im Mittelpunkt steht die Betrachtung von ‘Portalen` als Darstellung von real existierenden (und kulturell relevanten) Objekten in Form von virtuellen Interfaceelementen im Spiel. Diese digitalen Repräsentationen realer Objekte finden sich im öffentlichen Raum. Exemplarisch werden drei verschiedene Typen von «Portalen〉 diskutiert. 


\section{Portale als Informationsquellen für spielbezogene und reale Elemente}

Die Darstellung von Portalen in Ingress erfolgt über die Übersichtskarte des «Scanners» bzw. des jeweiligen Smartphones (Abb. 1). In Form von Science-Fiction orientierten 3D-Animationen werden in dieser Ansicht die Standorte der virtuellen Portale auf einer Karte, basierend auf der realen Welt, visualisiert. Die Darstellung zeigt zudem spezifische Zustände der Portale (z. B. die aktuelle Fraktionszugehörigkeit in Form unterschiedlicher Farben). Wird ein Portal auf dem Scanner angetippt, öffnet sich eine spezifische Darstellung des einzelnen Portals mit verschiedenen Elementen und möglichen Interaktionen (Abb. 2, links). Im oberen Drittel der Darstellung ist z. B. das Level des Portals angezeigt (hier: L7) sowie der Name und die Fraktion des/der aktuellen Portalbesitzers/-in (hier: Mammawutz der Fraktion «Resistance»). Andererseits werden in dieser Ansicht auch Bezüge zu den realen Objekten veranschaulicht. Dies erfolgt z. B. in Form eines (in der Regel von Spielern/-innen vergebenen) Namens (hier: «Karo 5») sowie in Form eines Fotos (in türkis hervorgehobene Box mit Foto und einem Informationssymbol [i]). Der Name «Karo 5» verweist einerseits auf die Bezeichnung des Gebäudes an der TU Darmstadt sowie andererseits auf die zugehörige Strasse und Hausnummer: Karolinenplatz 5.
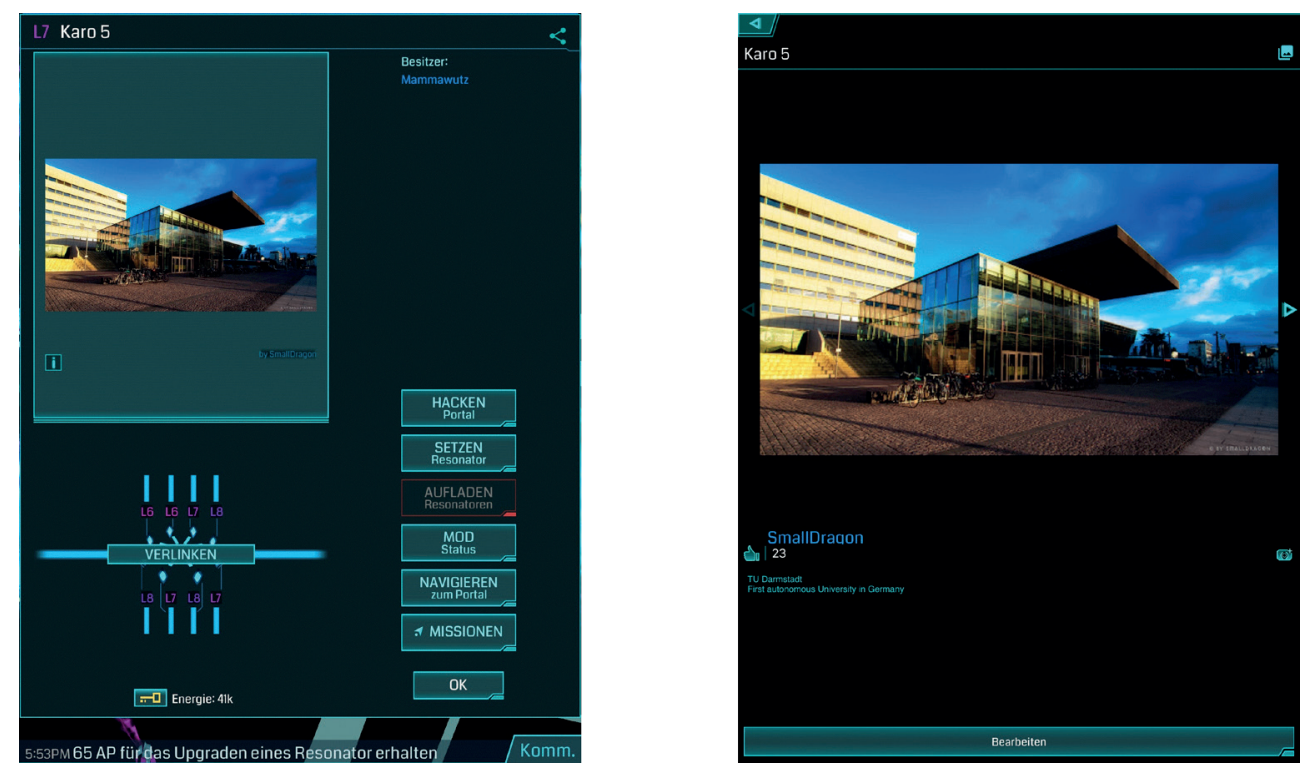

Abb. 2.: Portaldarstellung des «Karo 5» (links) sowie die Detailansicht des realen Objektes (rechts). Quelle: Screenshot des Spiels Ingress.

Eine weitere Ansicht von Portalen öffnet sich über das Antippen des Informationssymbols (Abb. 2 rechts). Diese beinhaltet den bereits bekannten Namen des Portals und eine vergrösserte Darstellung des zugehörigen Fotos. Der Spielername unter dem Foto gibt an, von wem das Foto eingereicht wurde. Neben der Möglichkeit das 
Bild zu 〈liken) und einer quantitativen Darstellung der bisherigen «Likes〉 wird eine von Spieler/innen erstellte Beschreibung zum Portal angezeigt: «TU Darmstadt. First autonomous University in Germany». Diese Beschreibung ist insofern interessant, da nicht das reale Objekt - das Gebäude mit dem Namen «Karo 5» - näher konkretisiert wird. Vielmehr erscheint das «Karo 5» symbolisch für die TU Darmstadt zu stehen. Der Hinweis auf die Autonomie der TU verweist über das reale Objekt hinaus auf eine bildungspolitische Entscheidung. Die im Spiel enthaltenden Informationen, die über das Spiel sowie über konkrete reale Objekte hinausgehen können, markieren erste mögliche Irritationsanlässe.

\section{Portale als Momentaufnahmen kultureller Objekte im öffentlichen Raum}

Ein weiteres Beispiel für ein Portal auf dem Campus der TU Darmstadt trägt den Namen «Der Dealer im Dunklen Durchgang» (Abb. 3, links). Dieses Portal beinhaltet die Beschreibung: «Im Durchgang zur Mensa der TU Darmstadt befindet sich ein kleines Stück alter Streetart, das erhaltenswert ist». In heutiger Betrachtung des realen Objektes zeigt sich, dass das künstlerische Objekt nicht mehr vorhanden ist (Abb. 3, rechts). Im Zuge der Renovierungsarbeiten wurde die Aussenfassade des Gebäudes erneuert und der «Dealer» entfernt. Dieses Portal ist insofern interessant, als dass die Abbildung des «Dealers» zum Bestandteil der Spielumgebung von Ingress geworden ist, obwohl das reale Objekt nicht mehr existiert. Diese beim Besuch des Portals erfahrbare Differenz zwischen der virtuellen Spielumgebung und der realen Welt kann Auslöser für Irritationen sein. Weitere Beispiele für ähnliche Portale finden sich insbesondere in Städten mit Ausstellungen im öffentlichen Raum die nur für einen begrenzten Zeitraum zugänglich waren, z. B. von der documenta in Kassel. So stellen verschiedene Ingress-Portale Momentaufnahmen öffentlicher Räume und realer Objekte dar und ermöglichen in diesem Sinne einen Blick in die Vergangenheit. Ohne ein entsprechendes Vorwissen ist zugleich die Lesart möglich, es handele sich bei dem virtuellen Portal um einen ‘Fake〉. 

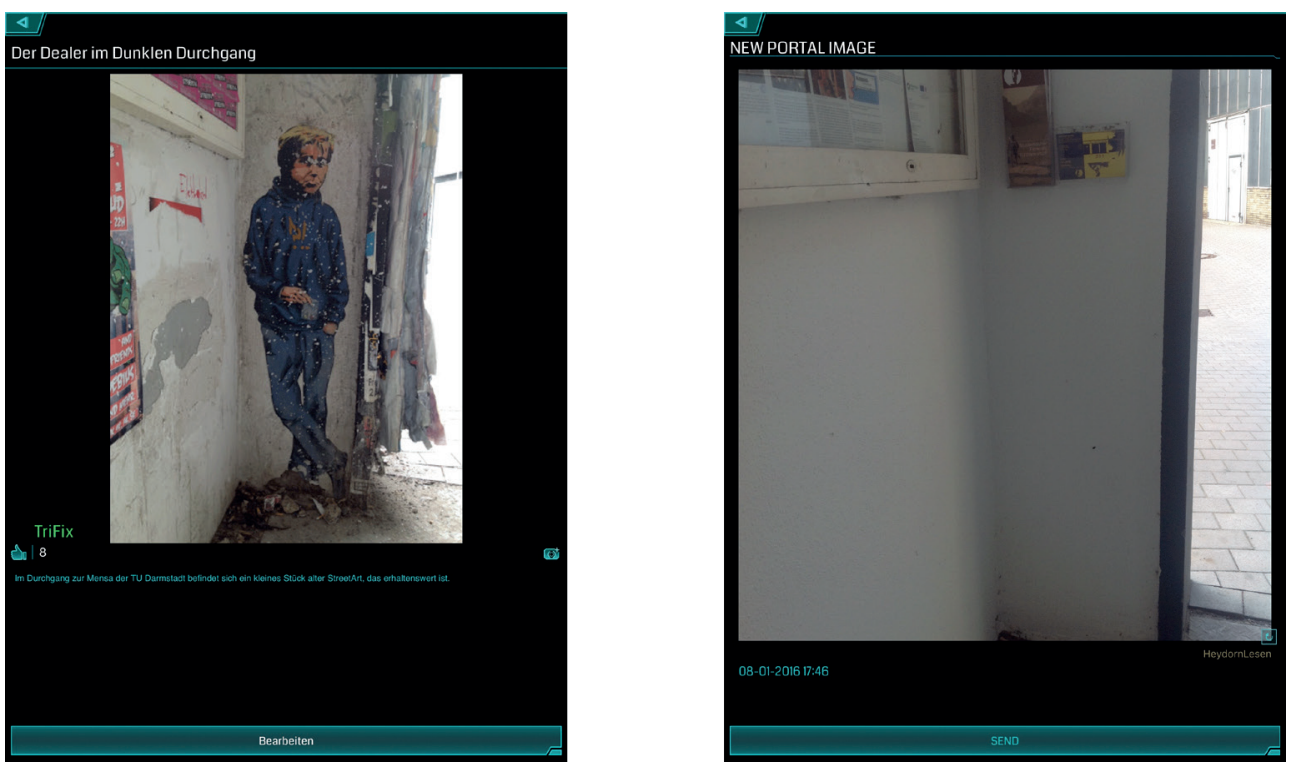

Abb. 3.: Detailansicht vom Portal «Der Dealer im Dunklen Durchgang» (links) und aktuelles Foto des Gebäudes (rechts). Quelle: Screenshot des Spiels Ingress.

\section{Portale mit ironischen Bezeichnungen und spielinternen Verweisen}

Als letztes Beispiel dienen zwei Portale, die Irritationen und Anlässe zur Reflexion auf unterschiedlichen Ebenen bieten können. Das erste Portal (Abb. 4) findet sich in einem Park in der Nähe des Campus Stadtmitte der TU Darmstadt. Das Foto markiert einen Schachtdeckel ohne auffällige Besonderheiten. Vielmehr finden sich weitere Schachtdeckel vom selben Bautyp in der näheren Umgebung ohne als Portal markiert zu sein. Unter Berücksichtigung der Narration des Spiels und der Erläuterung, dass es sich bei Portalen um kulturträchtige Orte oder Objekte handele, stellt sich hier durchaus die Frage, welche kulturelle Bedeutung dieser Schachtdeckel als reales Objekt besitzt. Vor diesem Hintergrund scheint es durchaus fragwürdig, warum (a) der Schachtdeckel als Portal eingereicht und (b) von Niantic als Portal zugelassen wurde. Der Vergleich des Fotos mit der Bezeichnung «Das Loch des Grauens» kann zu weiteren Fragen und Irritationen führen, z. B. worauf sich «das Grauen» an dieser Stelle beziehen könnte. Im Sinne von Fromme (2006) kann argumentiert werden, dass entsprechende Bezeichnungen eine Distanz zu medialen Repräsentationen des realen Objektes eröffnen. 

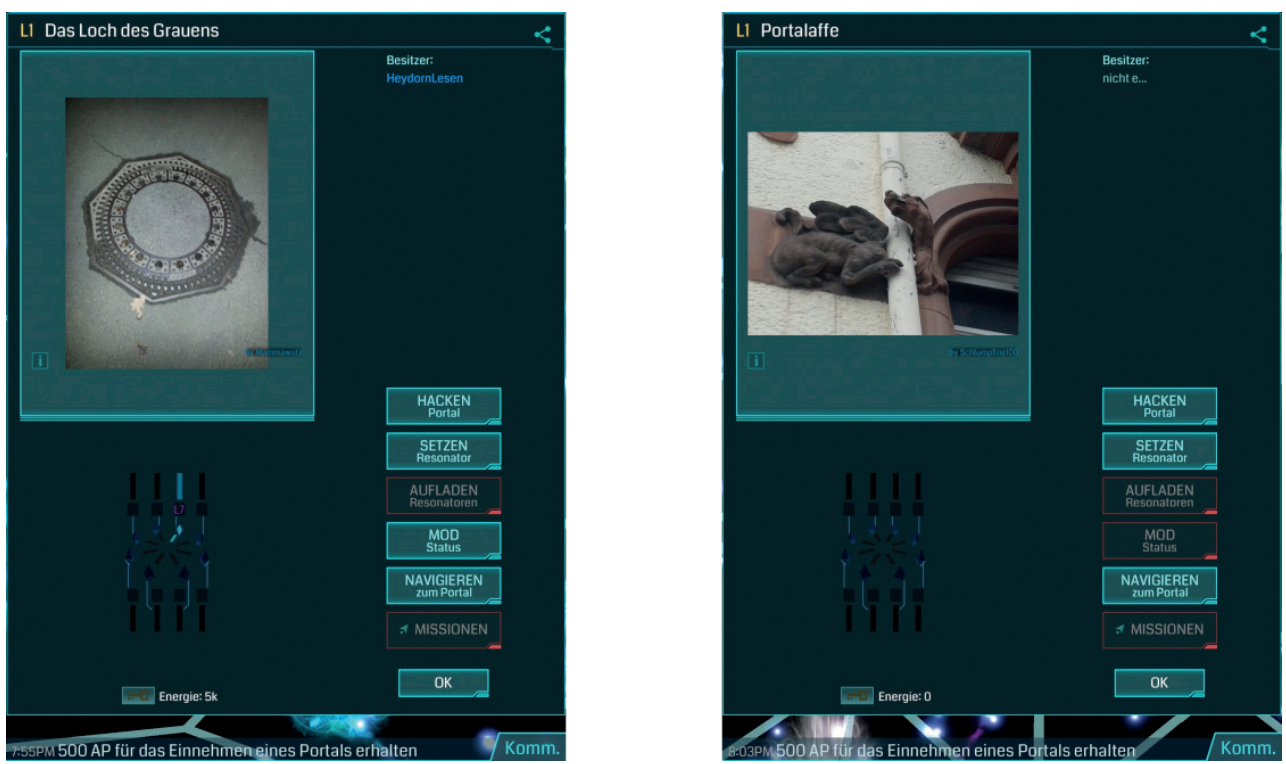

Abb. 4.: Portalansicht «Das Loch des Grauens» (links) und Portalaffe (rechts). Quelle: Eigener Screenshot des Spiels Ingress.

Portale wie das «Loch des Grauens» werden im Rahmen der Spielcommunity durchaus kontrovers diskutiert. Wenngleich die Möglichkeit besteht, Bearbeitungsvorschläge zur Umbenennung und Löschung von Portalen über das Spiel einzureichen, werden nicht alle dieser Vorschläge von Niantic zugelassen. Das zweite Portal mit dem Namen «Portalaffe» kann als Ausdruck der Unzufriedenheit der Spielcommunity verstanden werden, der den Umgang von der das Spiel anbietenden Firma Niantic mit Portaleinreichungen in ironischer Weise darstellt. So wird in der Spielcommunity die - nicht ganz ernst gemeinte - These vertreten, dass Portaleinreichungen bei Niantic nicht kriteriengeleitet von Menschen geprüft werden. Vielmehr erweckt der Begutachtungsprozess von Portalen den Eindruck, Affen würden eingereichte Portale quasi zufällig genehmigen bzw. ablehnen. Der Fakt, dass ein Portal mit dem Namen «Portalaffe» genehmigt wurde, obwohl das Foto keinen Affen im eigentlichen Sinne darstellt, wird von einigen Spielern/-innen gerne als Stütze ihrer These herangeführt. Ohne ein entsprechendes Vorwissen kann die Differenz zwischen dem Foto und der Namensgebung, die den Reviewprozess von Portalen in ironischer Weise markiert, nicht erschlossen werden.

\section{Zusammenfassung und Ausblick}

Augmented Reality Spiele wie Ingress stellen aktuell neue mediale Phänomene dar, die sich z. B. in der Verschränkung von virtuellen und realen Erfahrungsräumen als konstitutive Spielelemente zeigen. Eine erziehungswissenschaftliche Auseinandersetzung 
mit Ingress als potenziellen Kultur- und Bildungsraum ist bisher jedoch kaum erfolgt. Inwiefern die sich neu eröffnenden Optionen Lern- und Bildungsprozesse anregen können und welche Bedeutung die neuen Interaktionsmöglichkeiten für die Entwicklung von Online-Gaming-Kulturen haben, bleibt derzeit offen.

In erster Annäherung an die für diesen Beitrag relevante Fragestellung, inwiefern das Ingress-Spielen die Perspektive von Spieler/innen auf den öffentlichen Raum verändern kann, wurden die Erkenntnisse einer Interfaceanalyse skizziert. In der Betrachtung von (Portalen) als ausgewählte visuelle Darstellungen des Interfaces wurden verschiedene Elemente markiert, die potenziell Irritationen und Differenzerfahrungen ermöglichen: Portale, die (1.) weiterführende und/oder widersprüchliche Informationen für reale Objekte bieten, die (2.) Momentaufnahmen bereits nicht mehr existierender kultureller Objekte darstellen und die (3.) reale Objekte in ironischer Weise präsentieren. Mit der Fokussierung auf «Portale` ist dabei nur ein erster Einblick in das Spiel Ingress sowie in die Spielelemente von Augmented Reality Spielen präsentiert worden. Zugleich finden sich entsprechende Elemente auch über Ingress hinaus in weiteren Augmented Reality Spielen, z. B. in Form von Pokéstops beim Spiel PokémonGo. Eine weiterführende Untersuchung der in diesem Beitrag offen gebliebenen Fragen erfolgt derzeit auf Basis einer qualitativen Interviewstudie mit Ingress-Spieler/innen.

\section{Literatur}

Beil, Benjamin. 2013. Game Studies. Eine Einführung. Münster: LIT Verlag.

Breuer, Johannes, und Malte Elson. 2015. «Lernwerkzeug, Suchtmittel oder doch nur ein Spiel? Über die Wirkung von Computer- und Videospielen auf ihre Nutzer-/innen.» In Was wird hier gespielt? Computerspiele in Familie 2020, herausgegeben von Peter Holnick, 45-67. Opladen, Berlin u. Toronto: Verlag Barbara Budrich.

Chess, Shira. 2014. «Augmented Regionalism: Ingress as Geomediated Gaming Narrative.» Information, Communication \& Society 17(9), 1105-1117. doi:10.1080/1369118X.2014.881903.

Damberger, Thomas. 2016. «Augmented Reality als Bildungsenhancement?» Medienimpulse 2016/01. http://www.medienimpulse.at/articles/view/893.

Freyermuth, Gundolf S., Lisa Gotto, und Fabian Wallenfels. 2013. Serious Games, Exergames, Exerlearning. Bielefeld: Transcript Verlag.

Fromme, Johannes. 2006. «Zwischen Immersion und Distanz» In Clash of Realities. Computerspiele und soziale Wirklichkeit, herausgegeben von Winfried Kaminski und Manfred Lorber, 177-209. München: koaed.

Fromme, Johannes, Ralf Biermann, und Alexander Unger 2010. «Serious Games» oder «taking games seriouslys.» In Digitale Lernwelten. Konzepte, Beispiele und Perspektiven, herausgegeben von Kai-Uwe Hugger und Markus Walber, 39-57. Wiesbaden: VS Verlag für Sozialwissenschaften. 
Fromme, Johannes, Benjamin Jörissen, und Alexander Unger. 2008. «Bildungspotenziale digitaler Spiele und Spielkulturen.» MedienPädagogik 15/1, 1-23. doi:10.21240/ mpaed/15+16/2008.12.22.X.

Fromme, Johannes, und Christopher Könitz. 2014. «Bildungspotenziale von Computerspielen - Überlegungen zur Analyse und bildungstheoretischen Einschätzung eines hybriden Medienphänomens.» In Perspektiven der Medienbildung, herausgegeben von Winfried Marotzki und Norbert Meder, 235-286. Wiesbaden: Springer Verlag.

Ganguin, Sonja, und Anna Hoblitz. 2013. «Serious Games - Ernstes Spielen. Über das Problem Spielen, Lernen und Wissenstransfer.» In Serious Games, Exergames, Exerlearning, herausgegeben von Gundolf S. Freyermuth, Lisa Gotto und Fabian Wallenfels, 165-184. Bielefeld: Transcript Verlag.

Gotto, Lisa. 2013. "Serious Games. Einleitung.» In Serious Games, Exergames, Exerlearning, herausgegeben von Gundolf S. Freyermuth, Gundolf S., Lisa Gotto und Fabian Wallenfels, 139-144. Bielefeld: Transcript Verlag.

Grell, Petra, und Oliver Nuss. 2010. « Jetzt brauch ich 'n Raketenwerfer» - Differenzerfahrung und Irritation als Teil des Computerspielerlebens.» In Fokus Medienpädagogik - Aktuelle Forschungs- und Handlungsfelder, herausgegeben von Petra Bauer, Hannah Hoffmann und Kerstin Mayrberger, 221-238. München: kopaed.

Grell, Petra; Winfried Marotzki, und Heidi Schelhowe. 2010. Neue digitale Kultur- und Bildungsräume. Wiesbaden: VS Verlag für Sozialwissenschaften.

Gross, Friederike von, Winfried Marotzki, und Uwe Sander. 2008. Internet - Bildung - Gemeinschaft. Wiesbaden: VS Verlag für Sozialwissenschaften.

Kaminski, Winfried, und Martin Lorber. 2014. Spielwelt - Weltspiel. Narration, Interaktion und Kooperation im Computerspiel. München: kopaed.

Majorek, Marta, und Marta Du Vall. 2015. «Ingress: An Example of a New Dimension in Entertainment.» Games and Culture März 2015, 1-23. doi:10.1177/1555412015575833.

Niantic, Inc. 2013. Ingress. Android, iOS. Niantic, Google.

Pfannstiel, Jochen, Volker Sänger, und Claudia Schmidt. 2009. "Game-based Learning im Bildungskontext einer Hochschule - ein Praxisbericht.» MedienPädagogik 15/1, 1-21. doi:10.21240/mpaed/15+16/2009.04.07.X.

Stingeder, Karl H. 2013. "Googles Augmented-Reality-Game Ingress.» Medienimpulse April 2013/4. http://www.medienimpulse.at/articles/view/600.

The Pokémon Company, und Nintendo. 2016. PokémonGo. Android, iOS. Niantic.

\section{Abbildungen}

Abb. 1.: Ansicht der TU Darmstadt (Campus Mitte) durch den Scanner (links) sowie die Detailansicht des Portals «Karo 5» (rechts). Quelle: Screenshot des Spiels Ingress.

Abb. 2.: Portaldarstellung des "Karo 5» (links) sowie die Detailansicht des realen Objektes (rechts). Quelle: Screenshot des Spiels Ingress.

Abb. 3.: Detailansicht vom Portal «Der Dealer im Dunklen Durchgang» (links) und aktuelles Foto des Gebäudes (rechts). Quelle: Screenshot des Spiels Ingress.

Abb. 4.: Portalansicht «Das Loch des Grauens» (links) und Portalaffe (rechts). Quelle: Eigener Screenshot des Spiels Ingress. 